Tohoku J. Exp. Med., 2004, 204, 317-322

Case Report

\title{
Desmoplastic Medulloblastoma in a 48-Year-Old Male
}

\author{
İrfan Bayram, İbrahim İbíloĞlu, Serdar UĞraş, Nebí Yilmaz ${ }^{1}$ and Mustafa \\ HARMAN $^{2}$ \\ Department of Pathology, ${ }^{1}$ Department of Neurosurgery, and ${ }^{2}$ Department of \\ Radiology, 100. YIL University, Faculty of Medicine, Van, Turkey
}

\begin{abstract}
Bayram, İ., İbiloğlu, İ., UĞraş, S., Yilmaz, N. and Harman, M. Desmoplastic Medulloblastoma in a 48-Year-Old Male. Tohoku J. Exp. Med., 2004, 204 (4), 317-322 - Medulloblastoma is a malignant invasive embryonal tumor of the cerebellum with preferential manifestation in children. The peak of occurrence is seven years of age. Seventy percent of medulloblastomas occur in individuals younger than 16. In adulthood, $80 \%$ of medulloblastomas arise in the 21-40 years age group. A 48-year-old male patient was admitted to the hospital with complains of headache, ataxia, morning vomitting and difficulty in speech was operated with the diagnosis of presence of mass of $4 \times 7 \mathrm{~cm}$ size retaining a diffuse homogenous contrast in the posterior fossa. The diagnosis of desmoplastic medulloblastoma was given after histopathological examination. Immunohistochemical examination revealed that neoplastic cells showed staining with neuron-specific enolase and synaptophysin but not with glial fibrillary acidic protein. This lesion showed nodular, reticulin free-zones (pale islands) surrounded by densely packed, highly proliferative cells. The pale regions within the tumor did not contain reticulin fibers. Desmoplastic medulloblastoma is encountered especially in adulthood. This type of tumor rarely occurs beyond the fifth decade of life. We present a case of desmoplastic medulloblastoma in a 48-year-old male. - desmoplastic medulloblastoma; adulthood; posterior fossa

(C) 2004 Tohoku University Medical Press
\end{abstract}

Medulloblastoma is a tumor of neuro-epithelial origin that constitutes $4 \%-8 \%$ of all cerebral tumors. Approximately $70 \%$ of medulloblastomas is observed before the age of 16 years (Mazza et al. 1981; Tomita et al. 1986; Aron 1996). In adulthood, $80 \%$ of medulloblastomas arise in the
21-40 years age group. This tumor rarely occurs beyond the fifth decade of life. Classic and desmoplastic medulloblastoma are the most common histological variants. Classic medulloblastoma is composed of densely packed cells with round-tooval or carrot shaped highly hyperchromatic nu-

Received August 13, 2004; revision accepted for publication October 15, 2004.

Address for reprints: Dr. İrfan Bayram, Yüzüncü Yıl Üniversitesi Tıp Fakültesi Patoloji A.D., Maraş Caddesi Araştırma Hastanesi, 65200 VAN, Turkey.

e-mail: bayramirfan@yahoo.co.uk 
clei surrounded by scanty cytoplasm. Neuroblastic rosettes are a typical but not constant feature. Desmoplastic medulloblastoma shows nodular, reticulin-free zones (pale islands) surrounded by densely packed, highly proliferative cells that produce a dense intercellular reticuline fiber network. The nodules have reduced cellularity, a rarefied fibrillar matrix and marked nuclear uniformity. Nuclei of cells between nodules are usually more irregular and hyperchromatic (Giangaspero et al. 2000).

Medulloblastoma is rare in adults and is more frequently in the cerebellar hemisphere and more often DM than in children (Carrie et al. 1994; Malheiros et al. 2003). The adult presentation of medulloblastoma is an infrequent disease entity that shows both similarities and discrepancies with its pediatric counterpart. Adult medulloblastoma differs mainly in the incidence of more laterally located tumors and more desmoplastic histologic findings compared with pediatric tumors (Abacioglu et al. 2002).

Desmoplastic medulloblastoma is encountered especially in adulthood. This type of tumor rarely occurs beyond the fifth decade of life (Giangaspero et al. 2000; Malheiros et al. 2003). We present a case of desmoplastic medulloblastoma in a 48 -year-old male.

\section{Case Report}

A 48-year-old male patient applied to the Neurosurgery Policlinic of the Medical School of Yuzuncu Y1l University with complaints of 6 months history of headache, ataxia, morning vomitting, weakness, paresthesias, change in coordination and difficulty in speech. The patient was first physically examined, with findings of normal pupils. Further examinations revealed that Romberg and bilateral Babinski were both positive, nystagmus, dysmetria, and dysdiadochokinesia and there were disorders in the left cerebellar tests and an ataxic walk.

Magnetic resonance imaging (MRI) examination indicated a mass of $4 \times 7 \mathrm{~cm}$ size retaining a diffuse homogenous contrast in the posterior

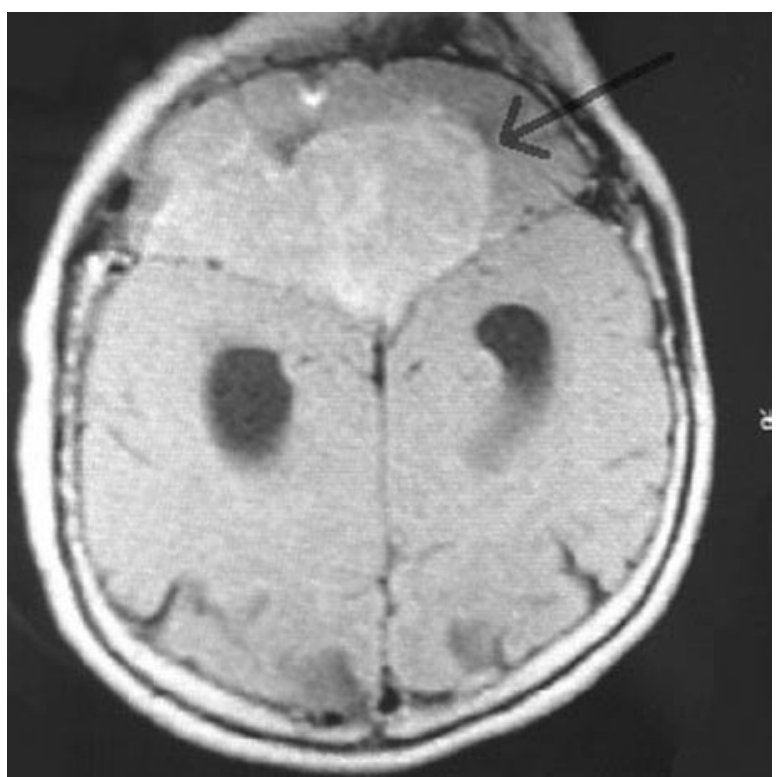

Fig. 1. Contrast-enhanced coronal T1-weighted MR image demonstrates diffuse contrast enhancement of the cerebellar mass lesion (arrow). Vermian mass lesion extends left cerebellar hemisphere and compresses fourth ventricle.

fossa, which compressed the ventricle and exerted a pressure on the cerebellum in the left suggestive medulloblastoma. The mass had hyperintense signal intensity on T2-weighted image and diffuse contrast enhancement on postcontrast T1weighted image (Fig. 1). Leptomeningeal seeding metastasis was not detected on the cerebral and spinal MRI investigations. Oncological staging (CT, total body, etc.) was negative for systemic tumors.

The case was operated upon these findings. During the operation, a bleeding mass in gray color was observed in the posterior fossa, which was aspirated easily. At surgery, subtotal excision of the tumor was performed and tumor was found to be attached brain tissue.

In macroscopic examinations, pink-beige tissue pieces of approximately $4 \mathrm{ml}$ volume with soft consistency were observed.

The samples for the histological and immunohistochemical examinations were fixed in $10 \%$ formalin, dehydrated in alcohol and then embedded in paraffin. Numerous sections were 


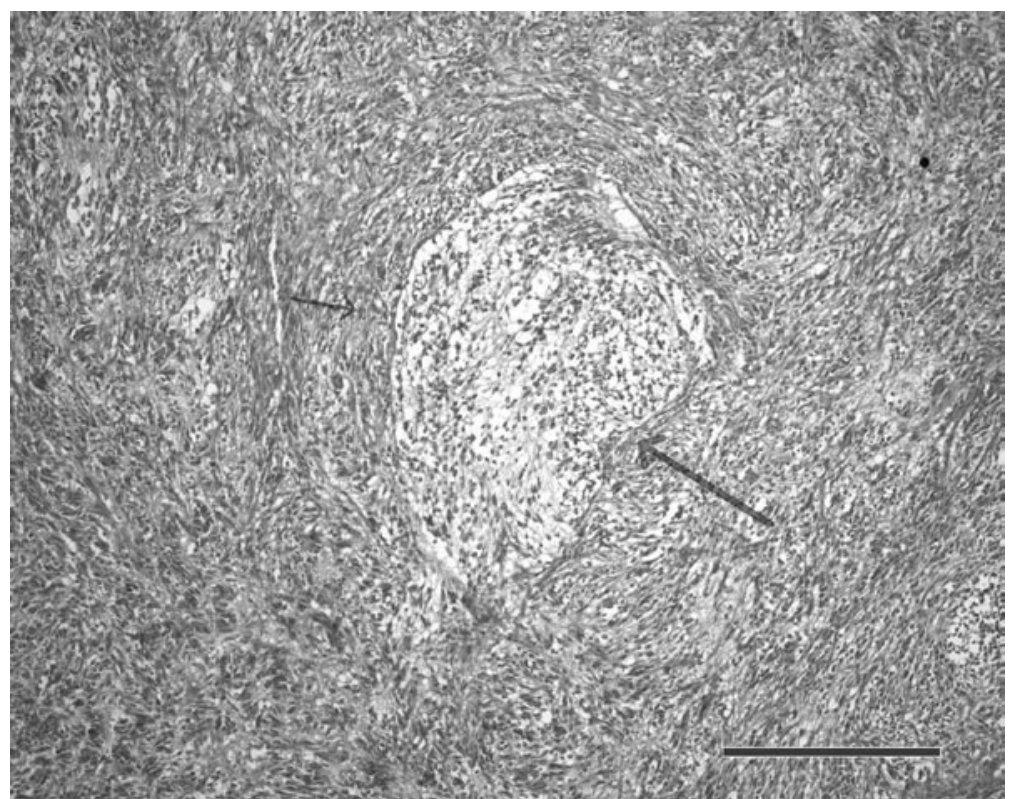

Fig. 2. Desmoplastic medulloblastoma. Nodules (long arrow) with reticuline-free zones, surrounded by densely packed, highly proliferative cells. Nuclei of cells between nodules were more irregular and hyperchromatic (short arrow). Scale bar, $1 \mathrm{~mm}$ (Haematoxylin-eosin stain, original magnification, $\times 100)$.

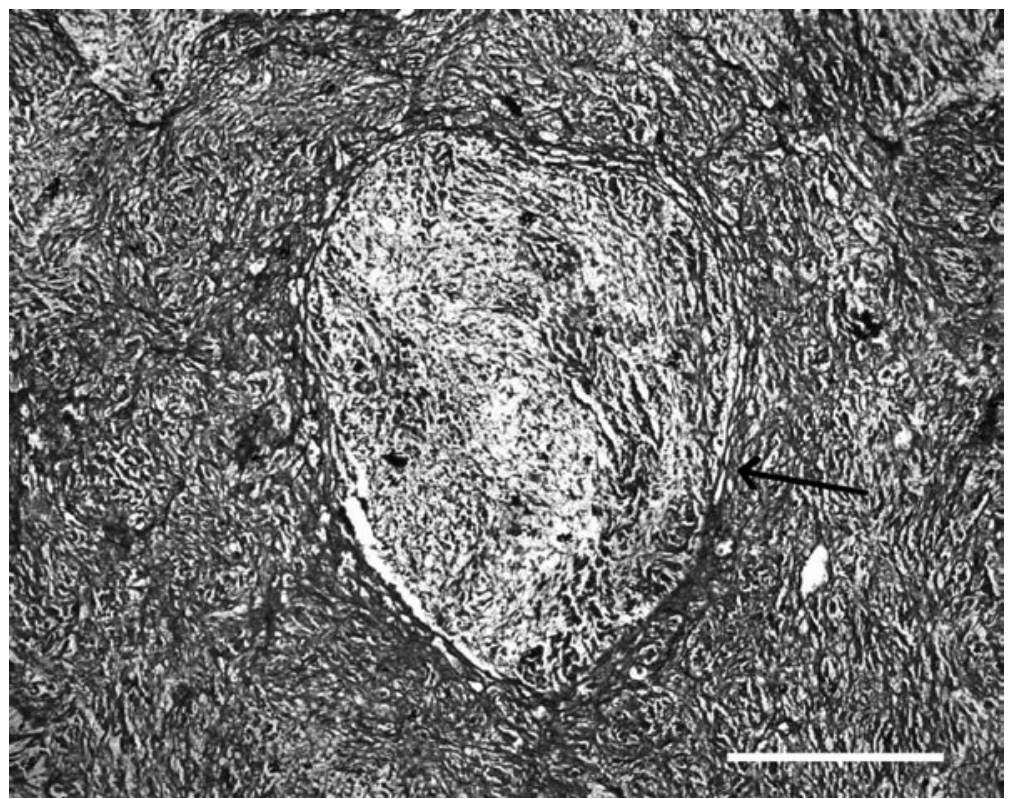

Fig. 3. Reticuline stain showed very cellular desmoplastic regions and a biphasic appearance with reduced reticuline fibers. There is not reticuline fibers in pale islands (arrow). Scale bar, $1 \mathrm{~mm}$ (Reticuline stain, original magnification, $\times 100$ ). 


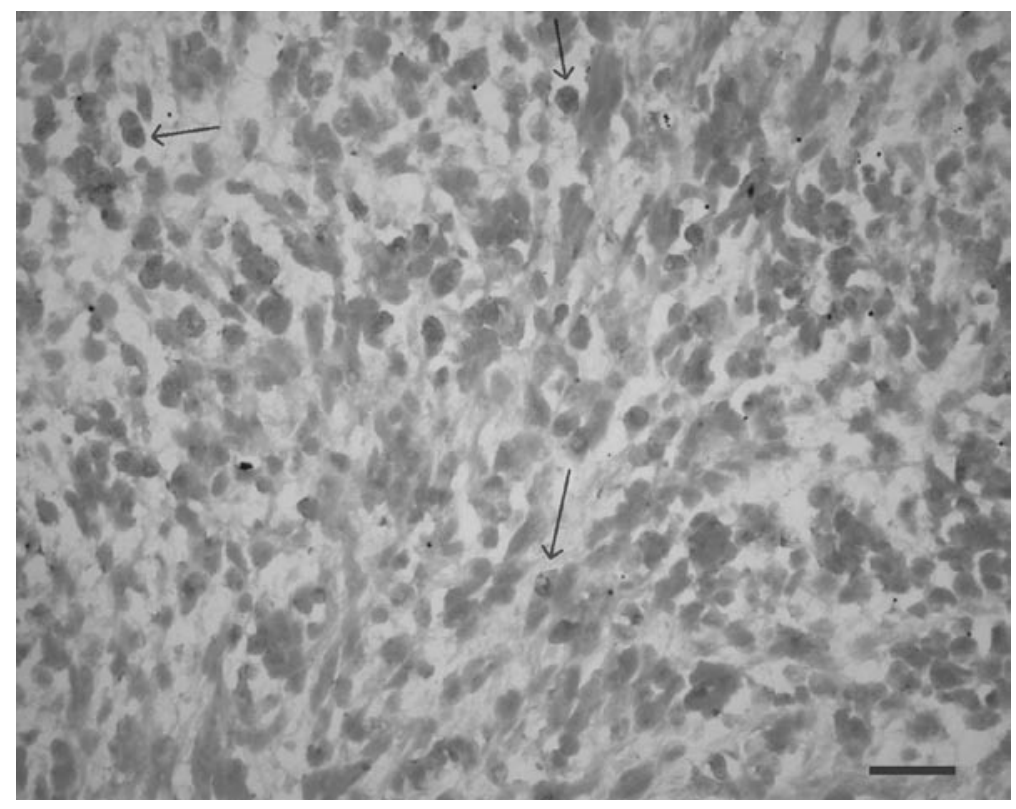

Fig. 4. Focal staining with NSE in tumor cells (arrows) with neuronal differentiation located within the islands. Scale bar, $0.1 \mathrm{~mm}$ (NSE stain, original magnification, $\times 400$ ).

stained with haematoxylin-eosin, reticuline stain, glial fibrillary acidic protein (GFAP), neuronspecific enolase (NSE) and synaptophysin. Histologic examination showed the characteristic features of a desmoplastic medulloblastoma. The tumor was composed of nodules with reticulinfree zones, surrounded by densely packed, highly proliferative cells (Fig. 2). These nodules had reduced cellularity and nuclear uniformity. Nuclei of cells between nodules were more irregular and hyperchromatic (Fig. 2). In histochemical studies, reticuline stain showed very cellular desmoplastic regions and a biphasic appearance with reduced reticuline fibers (Fig. 3). In immunohistochemical studies, NSE and synaptophysin showed that the tumor cells with neuronal differentiation located within the islands were focally stained (Fig. 4). GFAP indicated that neoplastic cells did not show any staining.

The patient made a good postoperative recovery. He was last seen at a follow-up examination 18 months after excision of the tumor and relatively asymptomatic. The patient was unable to receive adjuvant therapy due to some reasons.

\section{Discussion}

Medulloblastoma is a malignant, invasive embryonal tumor of the cerebellum with preferential manifestation in children. Medulloblastomas correspond histologically to WHO grade IV. Involvement of cerebellar hemispheres increases with the age of the patient. The presenting clinical manifestations include truncal ataxia, disturbed gait, intracranial hypertension secondary to obstruction of CSF-flow (cerebro spinal fluidflow) and lethargy, headache, and morning vomiting (Giangaspero et al. 2000).

Medulloblastoma has variable appearances on MRI in both children and adults. Adults are more likely to have heterogeneous cerebellar hemisphere tumors, and this is thought to be related to the greater prevalence of desmoplastic tumors in adulthood (Malheiros et al. 2003).

Koci et al. (1993) reviewed preoperative MRI of 12 patients aged 18-53 years, seen over a period of 9 years. Although they included five $\mathrm{DM}$, the only characteristics were signal intensity on T2-weighted images and superficial extension and it was suggested that there are no pathogno- 
monic MRI criteria for differentiating DM from CM.

Malheiros et al. (2003) reviewed preoperative MRI of 9 patients aged 23-39 and 53 years (53 years old one was CM). Only one tumor, a DM, was vermian. The cerebellar hemisphere tumors, including the three CM, one of which was bilateral, were paramedian in seven and lateral in one. Signal intensity was heterogeneous in all patients, predominantly low or isointense on $\mathrm{T} 1$ and predominantly isointense (though sometimes slightly high) on T2-weighted images, with a variable number of cystic or necrotic foci. All patients had extension to the surface of the cerebellum and only two had well-defined margins. Contrast enhancement was extremely variable, ranging from none in one DM to slight or intense in three CM and five DM. It was heterogeneous in seven, including three $\mathrm{CM}$, and homogeneous in one DM.

In the present case MR examination indicated a mass of $4 \times 7 \mathrm{~cm}$ size retaining a diffuse homogenous contrast in the posterior fossa, which compressed the ventricle and exerted a pressure on the cerebellum in the left suggestive medulloblastoma. The mass had hyperintense signal intensity on T2-weighted image and diffuse contrast enhancement on postcontrast T1weighted image.

The discovery of a medulloblastoma in an elderly patient raises several issues. The majority of cerebellar tumors in elderly patient are metastases (Rosenblum et al. 2000). In our patient, oncological staging was negative for systemic tumors.

Although cerebellar medulloblastomas are considered to be typically neoplasms of childhood, they are not particularly rare in adults whom between the ages of 25-45 years, the second peak age medulloblastoma, but very few reports exist for ages of 45 years or over (Salvati and Cervoni 2000; Malheiros et al. 2002; Sarkar et al. 2002). During our literature survey on medulloblastoma of adulthood whom older than 45, we have not come across any data, except 9 pa- tients (one of them DM) over 65 years of age reported in a study conducted by Salvati and Cervoni (2000) and a series of 15 patients between the ages of 23-48 years reported by Malherios et al. (2002).

Childhood tumors were more commonly of classical histology and midline location while the desmoplastic variant and lateral location occurred more frequently in adults. Adult medulloblastomas were biologically less aggressive (Carrie et al. 1994; Sarkar et al. 2002). In the present case tumor was of desmoplastic histology and midline location.

Long-term survival was not uncommon. Although chemotherapy may be useful and well tolerated, surgery and postoperative craniospinal radiotherapy are the mainstay treatments for adult medulloblastoma patients. Long-term follow-up is necessary, because late recurrences are not rare. Spinal seeding at presentation is poor prognostic factor for recurrence (Abacioglu et al. 2002; Malheiros et al. 2002).

\section{References}

Abacioglu, U., Uzel, O., Sengoz, M., Turkan, S. \& Ober, A. (2002) Medulloblastoma in adults: treatment results and prognostic factors. Int. J. Radiat. Oncol. Biol. Phsics., 54, 855-860.

Aron, B.S. (1996) Twenty-years experience with radiation therapy of Medulloblastoma. Am. J. Roentgenol., 105, 37-42.

Carrie, C., Lasset, C., Alapetite, C., Haie-Meder, C., Hoffstetter, S., Demaille, M.C., Kerr, C., Wagner, J.P., Lagrange, J.L. \& Maire, J.P. (1994) Multivariate analysis of prognostic factors in adult patients with medulloblastoma. Retrospective study of 156 patients. Cancer, $\mathbf{1 5}$, 2352-2360.

Giangaspero, F., Bigner, S.H., Kleihues, P., Pietsch, T. \& Trojanowski, J.Q. (2000) Medulloblastoma. In: Pathology and Genetics of Tumors of the Nervous System, edited by P. Kleihues \& W.K. Cavenee, IARC Press, Lyon, pp. 172-174.

Koci, T.M., Chiang, F. \& Mehringer, C.M. (1993) Adult cerebellar medulloblastoma: imaging features with emphasis on MRI findings. AJNR, 14, 929-939.

Malheiros, S.M., Franco, C.M., Stavale, J.N., Santos, 
A.J., Borges, L.R.R., Pelaez, M.P., Ferraz, F.A. \& Gabbai, A.A. (2002) Medulloblastoma in adults: a series from Brazil. J. Neurooncol., 60, 247-253.

Malheiros, S.M.F., Carrete, J.H., Stavale, J.N., Santos, A.J., Borges, L.R.R., Guimaraes, I.F., Pelaez, M.P., Franco, C.M.R. \& Gabbai, A.A. (2003) MRI of medulloblastoma in adults. Diagnostic Neuroradiol., 45, 463-467.

Mazza, C., Pasqualin, A., Dal Pian, R. \& Donati, E. (1981) Treatment of Medulloblastoma in children: long-term results following surgery, radiotherapy and chemotherapy. Acta Neurochirm., 57, 163-175.

Rosenblum, M.K., Bilbao, J.M. \& Ang, L.C. (2000) Neuromuscular system. In: Ackerman's
Surgical Pathology, edited by J. Rosai, eighth edition, Mosby-year book, Inc., St. Louis, Missouri, pp. 2227-2397.

Salvati, M. \& Cervoni, L. (2000) Medulloblastoma in late adults. Case report and critical review of the literature. J. Neurosurg. Sci., 44, 230-232.

Sarkar, C., Pramanik, P., Karak, A.K., Mukhopadhyay, P., Sharma, M.C., Singh, V.P. \& Mehta, V.S. (2002) Are childhood and adult medulloblastomas different? A comparative study of clinicopathological features, proliferation index and apoptotic index. J. Neurooncol., 59, 49-61.

Tomita, T. \& McLone, D.G. (1986) Medulloblastoma in childhood: results of radical resection and low-dose neuraxis radiation therapy. $J$. Neurosurg., 64, 238-242. 